\title{
Afasias e Áreas Cerebrais: Argumentos Prós e Contras à Perspectiva Localizacionista
}

\author{
Aphasias and Brain Areas: Positive and Negative Aspects \\ of the Localization Argument
}

\author{
Ana Cláudia C. Vieira ${ }^{*}, a, b$, Antônio Roazzi ${ }^{b}$, Bianca Manchester Queiroga ${ }^{b}$, \\ Rafaella Asforab ${ }^{\text {\& }}$ Marcelo Moraes Valença ${ }^{b}$ \\ ${ }^{a}$ Hospital da Restauração, Recife, Brasil \& ${ }^{b}$ Universidade Federal de Pernambuco, Recife, Brasil
}

\begin{abstract}
Resumo
O objetivo do presente estudo foi investigar como áreas corticais comprometidas por um acidente vascular encefálico (AVE) estão relacionadas com os diferentes tipos de afasia, visto que há controvérsias com relação à localização da lesão no cérebro e as características dos quadros de alteração da linguagem. Foram avaliados 26 indivíduos destros, portadores de lesão cérebro-vascular esquerda e de sintomas afásicos, através do protocolo de Montreal-Toulose, módulo standard inicial - versão alpha, que inclui provas de nomeação, repetição, compreensão oral, compreensão escrita e leitura, e de uma entrevista, que possibilitou a avaliação da fluência do discurso. Os sujeitos foram divididos em quatro subgrupos, de acordo com o sítio de lesão: frontal, temporal, têmporo-parietal e parietal/ parieto-occipital. Os dados foram submetidos a uma análise multidimensional (Similarity Structure Analysis [SSA]) e o método das variáveis externas como pontos. Os resultados revelaram uma correlação positiva alta entre lesão na área frontal e o acometimento da fluência no discurso, assim como correlações positivas altas entre lesão no lobo temporal e prejuízos em todas as habilidades avaliadas no teste: nomeação, repetição, compreensão oral, compreensão escrita e leitura, o que, até certo ponto, corrobora as idéias de estudos localizacionistas, na medida em que estabelece o papel preponderante do lobo temporal para a linguagem e a importância do lobo frontal para as praxias da fala. No entanto, nos demais subgrupos, têmporo-parietal e parietal/ parietooccipital, se observou correlações positivas apenas com a compreensão oral e com a repetição, essa última somente no segundo grupo e com índice baixo, e correlações negativas altas com a fluência no discurso, o que sugere que esta habilidade se manteve preservada nestes grupos e fragiliza o argumento localizacionista. Palavras-chave: Afasia; Avaliação da Linguagem; SSA.
\end{abstract}

\begin{abstract}
The aim of the present work was to investigate how cortex areas compromised by Cerebral Vascular Accident (CVA) are related to the classification of aphasia, since there are controversies about the location of the lesion in the brain and the characteristics related to variations in speech patterns. Through the Montreal-Toulose protocol, 26 right-handed persons who were affected by left cerebral-vascular lesion and have been showing aphasic symptoms have been evaluated. The Montreal-Toulose protocol, initial standard module - version alpha, includes nomination, repetition, oral comprehension, reading and writing comprehension tests as well as an interview, which allows an evaluation of the discourse fluency. The subjects were allocated into four sub-groups, according to the lesion sites: frontal, temporal, temporoparietal and parieto-occipital. For the analysis of the data, a Multidimensional Similarity Structure Analysis (SSA) was carried out along with an external variable method. The results show a high positive correlation between the lesion in the brain's frontal lobe and difficulties in the discourse fluency as well as a high positive correlation between the lesion in the brain's temporal lobe and hindrances in all abilities tested: nomination, repetition, oral comprehension, reading and writing comprehension. It is in accordance with localizational studies, since it highlights the fundamental role of the temporal lobe for the language and the importance of the frontal lobe for the speech praxis. However, in the remaining subgroups (temporo-parietal and parieto-occipital) there have been positive correlations only between oral comprehension and repetition, with the latter showing correlation only with the second group and presenting a low score. High negative correlations with the discourse fluency were observed. It suggests that this ability has remained preserved in those groups, which in turns weakens the localization argument.
\end{abstract}

Keywords: Aphasia; Language; Evaluation; Taxonomy; SSA.

* Endereço para correspondência: Universidade Federal de Pernambuco, Centro de Ciências Humanas, Rua Acadêmico Hélio Ramos, s/n, Cidade Universitária, Recife, PE, Brasil, CEP 50670-901. E-mails: ana.vieira.fono@gmail.com, roazzi@gmail.com, queirogabianca@gmail.co,rafaellaasfora@gmail.com e mmvalenca@yahoo.com.br 
As alterações da compreensão e/ou expressão da linguagem decorrentes de lesões no hemisfério cerebral esquerdo, denominadas “afasias” (Faroqi-Shah \& Thompson, 2007), têm sido amplamente estudadas desde o século XIX, entretanto muitas questões relacionadas a esses distúrbios ainda não foram totalmente elucidadas. Um dos principais pontos de discussão e controvérsia ainda atual é a relação da localização da lesão no cérebro e as características dos quadros de alteração da linguagem.

Em uma revisão sobre os correlatos neuroanatômicos das afasias, Damasio (1998) destaca que os primeiros estudos localizacionistas apontavam que lesões na região Sylviana esquerda, conhecida como área da linguagem, seriam as grandes responsáveis pelos quadros afásicos.

Segundo Basso (2000), no passado, as síndromes afásicas eram caracterizadas a partir da relação existente entre as diferentes formas de afasia e os diferentes sítios de lesão na clássica área da linguagem que é formada por quatro componentes principais: área de Broca, área de Wernicke, giro supramarginal e giro angular.

Estudos mais recentes também destacam a importância das áreas adjacentes à região Sylviana para o funcionamento da linguagem. Knaus, Bollich, Corey, Lemen e Foundas (2006), por exemplo, descrevem que as regiões perisilvianas também estão relacionadas com a função da linguagem e destacam o envolvimento de regiões posteriores, especialmente do lobo temporal e da área de Heschl, no comprometimento da compreensão e de regiões anteriores, especialmente da porção opercular e triangular do lobo frontal, no comprometimento da expressão da linguagem.

Uma das classificações para os transtornos afásicos mais divulgada é a classificação de Boston, na qual os diferentes quadros afásicos se distribuem em dois grupos principais, tendo como principal divisor a fluência do discurso que, na maioria das vezes, está associada à dicotomia das lesões cerebrais anteriores e posteriores. De acordo com essa classificação, a afasia de Broca, a afasia transcortical motora e a afasia global fazem parte do grupo das afasias não-fluentes, enquanto que a afasia de Wernicke, a afasia de condução, a afasia transcortical sensorial e a afasia anômica pertencem ao grupo das afasias fluentes (Goodglass \& Kaplan, 1984).

Assim como a fluência, a habilidade de repetição de palavras e frases também tem sido considerada no estabelecimento de outra divisão para os transtornos afásicos, que classifica as afasias em centrais, quando a repetição está comprometida e não centrais, quando a repetição está preservada. A divisão em afasia central e não-central devese a localização da lesão em relação à fissura de Sylvius. Ou seja, as afasias centrais envolvem as regiões circundantes da fissura de Sylvius: área de Broca, área de Wernicke e a substância branca subjacente a estas áreas, conhecida como fascículo arqueado. Enquanto que nas afasias nãocentrais as lesões estão mais à margem dessa fissura (Murdoch, 1997; Perea-Bartolomé, 2001).
As manifestações dos comprometimentos da linguagem encontradas nos quadros afásicos são inúmeras. Considerando os critérios de fluência e não fluência (Classificação de Boston), serão descritos, a seguir, os principais comprometimentos lingüísticos dos quadros afásicos.

Autores como Barraquer (2008) e Vendrell (2001) relatam que nas afasias não-fluentes as alterações podem ir desde uma supressão total da expressão verbal, até distúrbios menos graves. Pode ocorrer um discurso apenas com as emissões curtas como as estereotipias, que são segmentos lingüísticos com ou sem significado, emitidos repetidamente pelo indivíduo. Para Mansur (2003) e Miceli, Silveri, Romani e Caramazza (1989) também pode ser observada a preservação da linguagem automática ou a presença de um discurso agramático, ou seja, uma produção da linguagem reduzida na extensão e na complexidade gramatical. Hillis (2007) ressalta que também é possível haver prejuízo na melodia e agilidade de articulação, com diminuição de palavras por minuto. Em todos esses casos, o maior comprometimento ocorre na expressão da linguagem e está geralmente relacionado com lesões nas áreas cerebrais anteriores do hemisfério esquerdo.

De modo diferente, nas afasias fluentes o discurso pode se apresentar com produções extensas, porém complexas e sem sentido. Essas produções, muitas vezes, são formadas por jargão (segmento que não tem significado lexical), parafasias (substituição de um vocábulo por outro que tenha a mesma classe semântica ou uma estrutura fonológica semelhante), neologismos (criação de novas palavras, compreendidas dentro de um contexto) e perífrases (substituição do nome do objeto pela sua função, como exemplo: o sujeito ao invés de dizer pente, diz: "Serve para pentear").

Com o advento das técnicas de diagnóstico por imagem, como a tomografia computadorizada e ressonância magnética funcional, alguns estudos têm confirmado a correlação dos sintomas afásicos aos clássicos referenciais localizacionistas, incluindo as afasias de Broca, de Wernicke, de condução e global (Damasio, 1998), assim como reafirmam o envolvimento de regiões no hemisfério esquerdo, mais precisamente dos lobos temporal, parietal e região pré-frontal nos processos léxico-semânticos (Pereira, Reis, \& Magalhães, 2003).

Por outro lado, outros estudos também baseados em imagens, revelaram um grande número de quadros afásicos não correspondentes à clássica correlação anátomoclínica (Dronkers, 2000). Uma dessas descobertas foi a visualização de lesões nas estruturas subcorticais em tomografias e, posteriormente, nas ressonâncias magnéticas. Tais lesões, nos pacientes acometidos, resultavam em alterações da linguagem semelhantes aos quadros afásicos decorrentes das lesões corticais em hemisfério esquerdo. Para Jodzio, Gasecki, Drumm, Lass e Nyka (2003) há três explicações para que as lesões nas áreas subcorticais resultem em quadros afásicos: (a) essas es- 
truturas têm uma ação direta na função da linguagem; (b) ocorre devido a uma disrupção de circuitos subcorticaiscorticais; e (c) pode simplesmente ser associado com hipoperfusão.

Dronkers e Ogar (2004) destacam, ainda, a partir de evidências de suas pesquisas, que uma mesma função lingüística pode ativar áreas cerebrais distintas. Esta dissociação da taxonomia com as áreas corticais clássicas da linguagem também foi defendida por Demonet, Thierry e Cardebat (2005) quando afirmam o envolvimento de áreas que se encontram distantes das regiões citadas pelos estudiosos do século XIX.

Perea-Bartolomé (2001) destaca que, com o avanço das técnicas de diagnóstico e o aprofundamento dos estudos dos transtornos afásicos se tornou cada vez mais difícil a tarefa de enquadrar cada caso a uma determinada forma clínica. Para a autora, cada vez que se investigava um maior número de pacientes, maior era o número das afasias que não correspondiam às classificações existentes, pois os testes, com freqüência, revelavam a presença de mais de uma síndrome afásica no mesmo indivíduo, sendo prudente classificá-lo em afásico misto não-fluente ou afásico misto fluente.

A partir desta perspectiva, Perea-Bartolomé (2001), baseada na grande diversidade anatomofuncional existente e na utilidade do conhecimento detalhado da semiologia de cada caso para a reabilitação e integração social do indivíduo afásico, afirma que o estudo das afasias deveria primar pelas manifestações clínicas e neuropsicológicas do indivíduo, mais do que na localização da lesão.

Como pode ser observado, há, por um lado, um grupo de pesquisadores como Jodzio et al. (2003) que, apesar do advento de novas técnicas diagnósticas, continuam encontrando evidências que reforçam as idéias localizacionistas de séculos passados e, por outro lado, um grupo que destaca a dificuldade em se estabelecer correlações entre os transtornos afásicos e as classificações existentes (Basso, 2000; Dronkers \& Ogar, 2004).

Com o propósito de contribuir para essa discussão e com a perspectiva de realizar uma análise MDS (Similarity Structure Analysis [SSA]) e o método de variáveis externas como pontos, ainda não apresentada na literatura que investiga a correlação lesão cerebral e afasia, o objetivo deste trabalho foi verificar como as áreas corticais comprometidas, a partir de um acidente vascular encefálico (AVE), estão inter-relacionadas com os diferentes tipos de afasia.

De modo específico, o estudo pretendeu responder aos seguintes questionamentos: (a) Haverá uma correlação significativa entre áreas corticais lesionadas e as habilidades de linguagem? (b) Em caso afirmativo: será que todas as áreas se relacionam do mesmo modo ou haveria gradações nestas correlações? (c) Em caso afirmativo: qual estrutura relacional dessas habilidades lingüísticas e como estas variáveis se comportariam em uma análise MDS (SSA) e no método de variáveis externas como pontos?

\section{Método}

\section{Participantes}

Participaram do estudo 51 indivíduos, com distúrbio da linguagem decorrente de AVE em hemisfério esquerdo. Dos 51 pacientes, foram excluídos os que tinham lesões extensas que comprometiam, no mesmo indivíduo, áreas anteriores e posteriores do hemisfério esquerdo, lesões com comprometimento de áreas subcorticais concomitante às lesões corticais ou exclusivas e indivíduos com dominância manual esquerda. Dos 51 indivíduos, 26 pacientes destros, apresentavam lesões corticais únicas e foram subdivididos em quatro grupos de acordo com o sítio da lesão: Frontal (09), temporal (08), têmporoparietal (04) e parietal/ parieto-occipital (05).

Quanto à idade, apresentaram média de 52, $69 \pm$ 2,73, com mínima de 27 e máxima de 72 anos. Quanto à escolaridade, a média foi de 6,27 $\pm 0,99$ anos, entretanto, quatro indivíduos do grupo não sabiam ler, sem ter freqüentado a escola e a máxima foi de 18 anos de escolaridade. Mesmo com uma ampla faixa de escolaridade entre os participantes da pesquisa, os trabalhos desenvolvidos por Soares e Ortiz mostram que a lesão é mais impactante e determinante no desempenho dos sujeitos afásicos do que a escolaridade (Soares \& Ortiz, 2008). Por esta razão, optamos por incluir todos os indivíduos com lesões únicas na amostra, independente do nível de escolaridade.

Todos os participantes eram pacientes das enfermarias de Neurologia/ Neurocirurgia e do ambulatório de Fonoaudiologia do Hospital da Restauração em RecifePE, no período compreendido entre o mês de fevereiro de 2005 a dezembro de 2006, e apresentavam queixa de distúrbio da comunicação como seqüela de AVE.

O diagnóstico de AVE foi obtido a partir do exame clínico, realizado por médico neurologista, e confirmado por exames de imagens tomográficas do encéfalo.

\section{Procedimento}

A avaliação da linguagem foi realizada pela fonoaudióloga do hospital após o diagnóstico médico do AVE em um intervalo de 20 a 60 dias do ictus. Constou de uma entrevista com o paciente e seus familiares e da aplicação do protocolo de Montreal-Toulose versão Alpha. Esse teste foi inicialmente utilizado no Brasil em um estudo multicêntrico desenvolvido por Lecours et al. (1987a), no qual houve a participação de indivíduos residentes no estado de Pernambuco. Desta forma, o Protocolo apresenta características que se adéquam a realidade dessa população tais como: questões regionais e escolaridade.

Após uma seqüência de três estudos, Lecours e colegas (Lecours et al., 1987a, 1987b, 1988) consideram o valor empírico do protocolo, pois, entre outros aspectos, compararam com sucesso pacientes com lesão unilateral no hemisfério direito e esquerdo, sem que houvesse, entretanto, a pretensão da utilização como teste psicométrico.

Outro aspecto importante, que propiciou uma ampla utilização deste protocolo na prática clínica, refere-se ao 
tempo reduzido de aplicação, aproximadamente $30 \mathrm{mi}$ nutos, uma vez que, nos primeiros dias após a lesão cerebral, os indivíduos apresentam uma maior fatigabilidade, que pode interferir no desempenho das tarefas cognitivas (Vendrell, 2001).

Na entrevista foram coletados dados da história do paciente desde os primeiros sintomas do AVE e a evolução do quadro até o momento. Essa entrevista foi gravada e transcrita, o que possibilitou a análise por dois juízes independentes e treinados. Um dos objetivos principais dessa conversa entre terapeuta e paciente era investigar a capacidade de compreensão da linguagem verbal e a fluência do discurso, caracterizada por parâmetros como: linha melódica (entonação adequada, volume, duração e extensão das frases), ausência de esforço articulatório com boa velocidade de emissão. Todos esses aspectos permitiram aos investigadores classificar o discurso como "fluente" ou "não-fluente", conforme será descrito nos resultados. Já a coerência nas respostas, o uso de parafasias e outros elementos deformantes comuns ao discurso afásico, apesar de estarem presentes no discurso dos pacientes, não foram mensurados neste trabalho.

Para Perea-Bartolomé (2001), a fluência verbal é um dos critérios a ser investigado para classificar as afasias, respeitando o binômio fluência e não-fluência. Esta investigação pode ser realizada na conversação e os parâmetros de avaliação, são a extensão das frases e o ritmo verbal.

Após a entrevista, foi realizada a avaliação da linguagem propriamente dita, através do protocolo de Montreal-Toulose versão Alpha. O teste foi elaborado para ser realizado em tempo reduzido de aproximadamente 30 minutos, podendo ser utilizado à beira do leito. O protocolo consta de pranchas com gravuras em preto e branco. As pranchas avaliam as seguintes habilidades lingüísticas: compreensão oral (11 pranchas), compreensão escrita (11 pranchas), leitura (11 pranchas), nomeação (12 pranchas) e repetição (oito palavras e três frases). O protocolo ainda contém tarefas de ditado e cópia, que neste estudo, não foram avaliados.

Para tanto, os desempenhos dos indivíduos afásicos na entrevista (fluência do discurso) e nas tarefas verbais do teste Montreal-Toulose versão Alpha (compreensão oral, compreensão escrita, leitura, nomeação e repetição) foram tomadas como variáveis de conteúdo e as áreas lesadas no hemisfério cerebral esquerdo (frontal, temporal, têmporo-parietal e parietal/ parieto-occipital) foram tomadas como variáveis externas. A técnica das variáveis externas como pontos foi utilizada para verificar a relação da variável externa com as tarefas de linguagem. Esta técnica propicia a integração de sub-populações nos mapas MDS, ou seja, permite localizar espacialmente variáveis externas como pontos na estrutura interna representada na projeção SSA que permanece inalterada. Deste modo, ao invés de se analisar diferentes mapas SSA, um para cada subgrupo, é construído um único mapa que representa, ao mesmo tempo, o desempenho nas tarefas de linguagem e as áreas cerebrais lesadas.

\section{Analise de Dados}

O desempenho dos participantes no protocolo Montreal-Toulose versão Alpha foi colocado em forma numérica, de acordo com o número de acertos em cada item de cada prova, conforme os critérios definidos por Lecours et al. (1987a).

Os dados foram analisados através de uma análise MDS (SSA) e o método de variáveis externas como pontos. Esta análise processa uma matriz de correlação entre $n$ variáveis, produzindo uma representação geométrica dos dados, usualmente em um espaço Euclidiano, de dimensionalidade mínima capaz de representar de forma fidedigna a relação entre todas as variáveis estudadas. Desta forma, as variáveis (no caso do presente estudo, a fluência e as habilidades lingüísticas avaliadas pelo protocolo e as áreas corticais lesionadas) são representadas graficamente como pontos em um espaço, podendo-se verificar a existência de estruturas relacionais, visto que a localização dos pontos neste espaço determinará a sua relação com os outros pontos, ou seja, com as outras variáveis, de modo que quanto maior for a correlação entre duas variáveis, mais próximos os pontos irão se localizar no mapa e vice-versa (Guttman, 1968; Young, 1987).

\section{Resultados}

Os resultados serão apresentados, inicialmente, através de uma matriz de correlação das variáveis conteúdos (fluência do discurso e as habilidades de linguagem avaliadas através do protocolo de Montreal-Toulose versão Alpha), com as variáveis externas (territórios das lesões sub-divididos em 4 grupos: frontal, temporal, têmporoparietal e parietal/ parieto-occipital (Tabela 1).

No que se refere às variáveis de conteúdo, é possível observar que a variável leitura se correlacionou fortemente com todas as demais variáveis avaliadas pelo teste, sendo que a correlação positiva mais alta foi com a compreensão oral e com a repetição $(0,86)$, seguidas de uma correlação positiva alta com a nomeação $(0,80)$ e compreensão escrita $(0,79)$ e de uma correlação positiva mais baixa com o discurso $(0,54)$. Correlações negativas, mesmo baixas, foram observadas somente entre a variável discurso e as variáveis repetição $(-0,14)$ e compreensão oral $(-0,03)$.

No cruzamento das variáveis externas (territórios de lesão) com as variáveis de conteúdo (fluência do discurso e habilidades lingüísticas avaliadas pelo protocolo), observou-se que a região frontal apresentou uma correlação positiva alta com a fluência do discurso $(0,90)$ e negativa alta com compreensão oral $(-0,86)$. Estes resultados indicam que a lesão nesta região implica em um grave comprometimento da fluência do discurso e um baixo comprometimento da compreensão oral.

Foi possível observar, também, que a região temporal se correlacionou positivamente com a maioria das tarefas de linguagem, com exceção da fluência no discurso $(-0,46)$, sendo mais altas as correlações com a nomeação $(0,76)$ e com a compreensão escrita $(0,71)$. 
Tabela 1

Matriz de Correlação (coeficiente de monoticidade) entre as Variáveis de Conteúdo e a Correlação das Mesmas com as Variáveis Externas

\begin{tabular}{|c|c|c|c|c|c|c|}
\hline Variáveis & 1. Discurso. & 2. Comp. oral & 3. Repetição. & 4. Nomeação. & 5. Comp. Escrita & 6. Leitura \\
\hline \multicolumn{7}{|l|}{ Variáveis de Conteúdo } \\
\hline 1. Discurso (fluência) & - & & & & & \\
\hline 2. Compreensão oral & $-0,03$ & - & & & & \\
\hline 3. Repetição & $-0,14$ & 0,77 & - & & & \\
\hline 4. Nomeação & 0,15 & 0,63 & 0,73 & - & & \\
\hline 5. Compreensão escrita & 0,69 & 0,84 & 0,60 & 0,31 & - & \\
\hline 6. Leitura & 0,54 & 0,86 & 0,86 & 0,80 & 0,79 & - \\
\hline \multicolumn{7}{|l|}{ Variáveis Externas } \\
\hline 7. Frontal & 0,90 & $-0,86$ & $-0,48$ & $-0,60$ & $-0,49$ & 0,90 \\
\hline 8. Temporal & $-0,46$ & 0,54 & 0,43 & 0,76 & 0,71 & 0,44 \\
\hline 9. Temporo-parietal & $-0,97$ & 0,24 & $-0,03$ & $-0,42$ & $-0,52$ & $-0,60$ \\
\hline 10. Parieto-Occipital & $-0,96$ & 0,66 & 0,14 & $-0,52$ & $-0,10$ & $-0,40$ \\
\hline
\end{tabular}

Por outro lado, lesões temporais mais abrangentes, aqui denominadas por têmporo-parietais, apresentam correlações diferentes em comparação com as lesões restritas à área temporal acima descrita, uma vez que foram observadas correlações negativas com todas as tarefas, com exceção da compreensão oral, onde se observou uma correlação positiva baixa $(0,24)$. Vale ressaltar a correlação negativa alta com o discurso $(-0,97)$, o que indica a preservação da fluência verbal neste quadro.
No caso das lesões parietais/ parieto-occipitais foi verificado um envolvimento maior (correlação positiva) com as tarefas de compreensão oral $(0,66)$ e de repetição $(0,14)$, essa última não muito alta. Além disso, também foi verificada uma alta correlação negativa com o discurso $(-0,96)$.

A fim de se realizar um escalonamento muldimensional dos resultados, através da representação geométrica, os dados foram submetidos a uma Análise da Estrutura de Similaridade (SSA), que está apresentada a seguir.

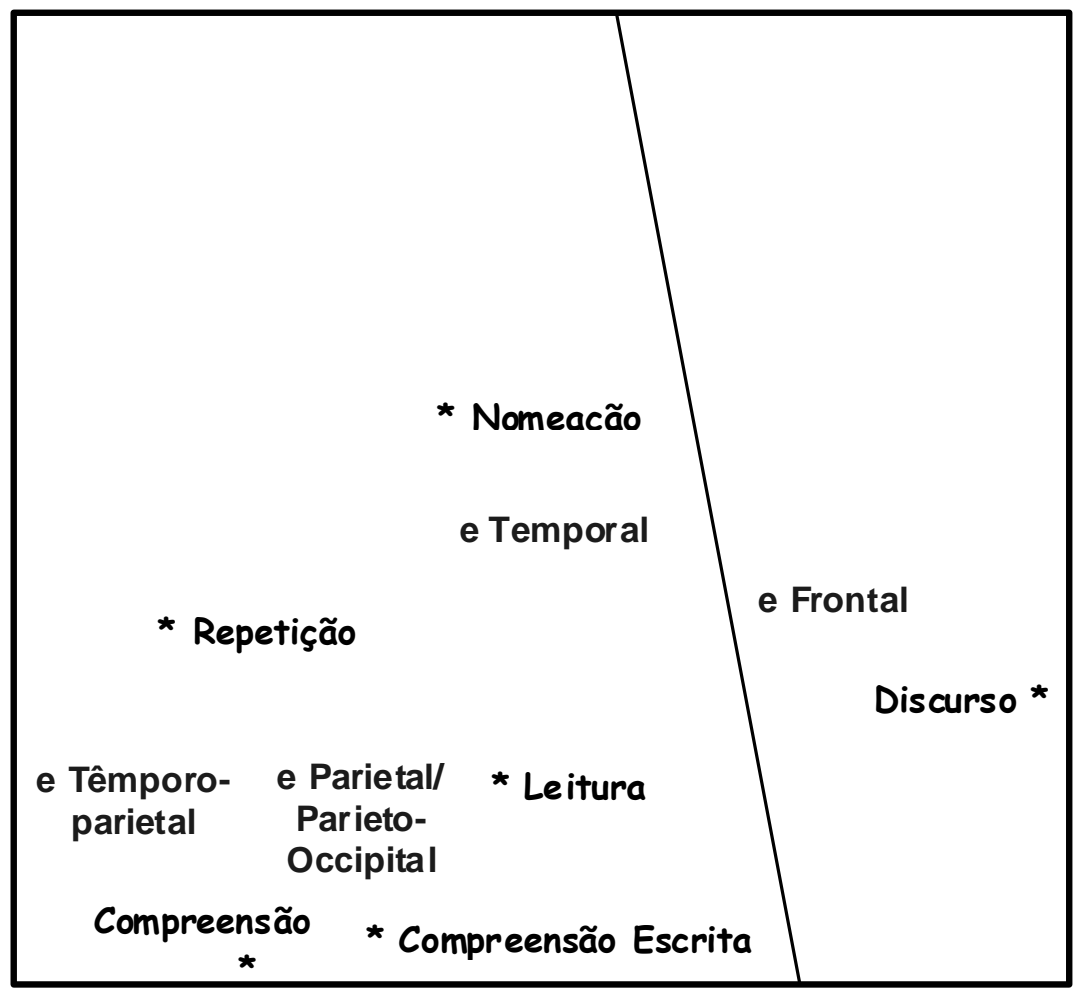

Figura 1. Análise SSA da interrelação entre as tarefas de linguagem do teste de Montreal-Toulose (variáveis de conteúdo “*”) e as lesões corticais no hemisfério esquerdo (variáveis externas “e”). Nota. Coeficiente de Alienação - 0,01; Projeção bidimensional. 
Como é possível observar na Figura 1, o método de variáveis externas como pontos demonstrou a importância da área temporal para a função da linguagem, tendo em vista a projeção central que esta área ocupa em relação às habilidades lingüísticas avaliadas.

Esta variável externa encontra-se no centro, em proximidade com as variáveis de conteúdo: repetição, nomeação e leitura, estando um pouco afastada das variáveis de conteúdo: compreensão oral e compreensão escrita.

Outro aspecto a ser destacado é a proximidade da variável externa frontal com a variável de conteúdo discurso, que por sua vez está mais distante das outras variáveis externas. Esta configuração permite visualizar, mais claramente, as relações entre as variáveis externas e de conteúdo já apontadas na Tabela 1.

Também é possível observar a proximidade das variáveis externas têmporo-parietal e parietal/parieto-occipital. É possível que esta proximidade seja decorrente da influência da área parietal sobre o funcionamento da linguagem.

\section{Discussão}

Com a proposta de lançar, mais uma vez, o questionamento quanto à existência de correlação significativa entre as áreas corticais e as habilidades de linguagem, este trabalho ratifica, parcialmente, a literatura que relata a existência de uma relação positiva entre a localização da lesão e o comprometimento afásico.

Gold, Balota, Kirchhoff e Buckner (2005), por exemplo, destacam que em determinados trabalhos esta relação é bastante seletiva, evidenciando particularidades das funções lingüísticas em áreas cerebrais cada vez mais delimitadas. Entretanto, esta correspondência nem sempre é preponderante.

Neste estudo, a evidência de uma redução na fluência do discurso relacionada com as lesões frontais foi confirmada. Entretanto, outros achados, como a ausência de correlação positiva da nomeação e da repetição, revelou quadros mais amenos, do que os comprometimentos citados pela literatura para as afasias decorrentes de lesões frontais, que muitas vezes são classificadas como afasia de Broca.

Um dos questionamentos que surge a partir dos resultados do presente estudo é porque os indivíduos com lesão frontal tiveram um melhor desempenho do que os indivíduos com lesão temporal na tarefa de nomeação do teste aplicado.

Já foi comprovado que lesões de áreas anteriores e grandes áreas posteriores parecem comprometer o acesso ao léxico em sujeitos afásicos, tornando-os vulneráveis a manifestação da anomia (Soares \& Ortiz, 2008). Entretanto, no presente estudo a mensuração da anomia só foi realizada a partir do escore da tarefa de nomeação do protocolo, ou seja, a partir da nomeação de gravuras de objetos em preto e branco.
Para DeLeon et al. (2007), a habilidade de nomear objetos, gravuras ou cenas é um processo complexo que envolve um número relativo de representações mentais e processos cognitivos. Para que o indivíduo nomeie um objeto é preciso uma série de atividades cognitivas, tais como: conhecer o significado do objeto, ter acesso ao significado específico daquele objeto e não apenas da classe semântica a que ele pertence, conhecer a cadeia fonológica correspondente à palavra que representa 0 objeto e ter a programação motora do planejamento articulatório para expressar a palavra correspondente ao objeto. Embora essas funções possam não ser completamente segregadas na anatomia do cérebro, elas podem estar comprometidas individualmente por lesões cerebrais (DeLeon et al., 2007).

No presente estudo, os pacientes com lesão frontal nomearam melhor as gravuras do que os pacientes com lesão temporal. Este dado corrobora os resultados observados por Murtha, Chertkow, Beauregard e Evans (1999), que através da utilização de ressonância magnética funcional, durante a nomeação de gravuras em sujeitos não lesionados, observaram uma maior ativação do giro fusiforme esquerdo e do giro temporal inferior esquerdo, além de áreas occipitais responsáveis pela recepção do estimulo visual e da área de Wernicke, para a decodificação do significado e acesso ao léxico. Entretanto, este dado por si só, não pode ser tomado como evidência de que os pacientes com lesão frontal tenham um menor comprometimento da nomeação do que os pacientes com lesão temporal.

Para Jodzio et al. (2003) os erros de nomeação de gravuras, observados nos pacientes, variam com relação a área lesionada. De acordo com os autores, a anomia também foi mais evidente nos pacientes com lesões cerebrais posteriores, além das lesões em estruturas subcorticais. Por outro lado, os pacientes com lesão na região frontal apresentaram erros de nomeação por terem dificuldades na percepção das gravuras, como também por apresentarem perseveração em suas respostas.

A anomia é uma inabilidade em recuperar o objeto ou a palavra, quer seja no nível fonológico ou na forma ortográfica, sem ter comprometido o conhecimento do seu significado (DeLeon et al., 2007). Esta alteração não se manifesta apenas na confrontação com objetos ou gravuras, como é avaliado no protocolo de Montreal e em tantos outros testes de afasias. Esta alteração muitas vezes se revela na dificuldade de evocar determinada palavra, na presença de hesitações do discurso, como também na ausência de uma fluência do discurso, caracterizando uma dificuldade clara de acessar o léxico. Assim, uma limitação da presente investigação é que não se mensurou as tentativas de evocação e de hesitações no discurso dos participantes.

Outra justificativa para um melhor desempenho dos pacientes com lesão frontal na tarefa de nomeação pode ser encontrada a partir das considerações do estudo rea- 
lizado por Shapiro, Môo e Caramazza (2006) que investigou as áreas cerebrais responsáveis pela emissão de verbos e substantivos em indivíduos normais. Este estudo revelou uma maior ativação da região pré-frontal esquerda e do lobo parietal superior esquerdo durante a emissão de verbos, enquanto que na emissão de substantivos a área mais ativada foi o giro temporal inferior esquerdo. Hillis (2007) corroborou com esses achados argumentando que os pacientes com afasia de Broca (lesão frontal) têm maior dificuldade na nomeação de verbos ao contrário dos pacientes com afasia de Wernicke (lesão temporal), que apresentam maior dificuldade em nomear substantivos. Assim, como a tarefa de nomeação do teste de Montreal consiste apenas na nomeação de 12 substantivos de grande freqüência, é possível que o desempenho dos pacientes com afasia por lesão frontal esquerda tenha sido melhor que o dos pacientes com lesão temporal esquerda por não haver a solicitação para a nomeação de outras classes gramaticais, especialmente os verbos.

Outra explicação seria que os participantes com lesão frontal esquerda do presente estudo tinham uma lesão mais restrita no córtex frontal do que o que tem sido reportado na literatura para evidenciar a afasia de Broca. Dronkers, Plaisant, Iba-Zizen e Cabanis (2007), por exemplo, argumentam que vários estudos posteriores a Broca explicam que a afasia nesta região é causada por grandes lesões que abrangem não apenas a área de Broca, mas também o córtex frontal circunvizinho, a substância branca subjacente, a ínsula, os gânglios da base e parte anterior do giro temporal superior. Isto implica que outras regiões do cérebro participam na produção de discurso além da área de Broca. Estes estudos revelaram que a área determinada por Broca, no século XIX, não causaria a síndrome clássica da afasia que leva seu nome, mas resultaria em déficits mais suaves da linguagem, porém sem causar uma completa redução na produção do discurso.

Por outro lado, os estudos de Alexander, Naeser e Palumbo (1990) reforçam a existência de uma variedade de síndromes da linguagem decorrentes de envolvimento do lobo frontal.

No presente estudo, apenas a redução no discurso foi uma característica marcante nos indivíduos com lesões frontais esquerdas, diferenciando-o nitidamente dos outros três grupos. Este perfil assemelha-se aos quadros das afasias transcorticais motoras da classificação de Boston (Goodglass \& Kaplan, 1984).

Outro resultado muito importante da presente pesquisa foi a observação da relação intrínseca das habilidades de linguagem avaliadas pelo protocolo com as lesões na área temporal esquerda, uma vez que, com exceção da fluência no discurso, os indivíduos com este tipo de lesão apresentaram prejuízos nas habilidades de compreensão oral e escrita, leitura, repetição e nomeação, observadas através das correlações positivas.

Vale salientar que, no presente estudo, a fluência do discurso foi analisada com base em características como velocidade, agilidade e comprimento das frases, não sendo contemplado o conteúdo e a coerência do discurso, como também a presença de elementos deformantes da linguagem como neologismos, parafasias e/ou jargões, que poderiam também definir um envolvimento da região temporal neste aspecto. De fato, a agilidade e velocidade articulatória geralmente encontram-se preservadas nos pacientes com lesão temporal (Goodglass \& Kaplan, 1984; Hillis, 2007; Perea-Bartolomé, 2001). Desta forma, ao que parece, a região temporal está intrinsecamente relacionada com a função da linguagem como um todo, em maior ou em menor grau, dependendo da tarefa analisada.

Diante do exposto é possível afirmar que os resultados do presente estudo corroboram com a idéia da correlação da área temporal com a sintomatologia descrita como característica da afasia de Wernicke, como afirmam Goodglass e Kaplan (1984).

Outro aspecto importante do estudo foi a correlação positiva da nomeação apenas com a região temporal. Dados da literatura, como os de Jodzio et al. (2003), confirmam que a nomeação nos quadros de lesão anterior, como a região frontal, se caracteriza muito mais como uma perseveração do estímulo anterior do que uma dificuldade própria de nomear os objetos. Já nas lesões posteriores, como na região temporal, o erro da nomeação seria caracterizado por emissões com parafasias, neologismos ou perífrases, revelando uma dificuldade clara de nomear os objetos. Esta característica foi observada na amostra do presente estudo. Os pacientes com lesão frontal, que conseguiram nomear no teste, apresentaram erros de perseveração ou presença de parafasia fonêmica, ou seja, o paciente produz sílabas na ordem errada ou distorce suas palavras com sons não intencionais. Enquanto que, os pacientes com lesão na região temporal demonstraram uma maior dificuldade de nomear, apresentando respostas longas, descrevendo o estímulo (circunlóquio), apresentando a funcionalidade do estímulo (perífrase), nomeando palavras que pertenciam a mesma categoria semântica do estímulo (parafasia semântica), mas demonstrando uma enorme dificuldade de selecionar a palavra alvo.

Este dado confirma a importância da região temporal esquerda para a nomeação, corroborando com outros estudos que afirmam haver prejuízo na inibição da ativação lexical decorrente de lesões nesta região, ou seja, haveria uma nítida dificuldade na seleção correta da palavra apropriada para determinado estímulo. Esta dificuldade pode ser observada na evocação de um elemento específico como na nomeação de objetos e gravuras (Hillis, 2007).

Por outro lado, os pacientes com lesão têmporo-parietal do estudo apresentaram um desempenho nas habilidades da linguagem melhor do que o grupo com lesão temporal única. Neste grupo de quatro indivíduos foi observado um prejuízo maior apenas na habilidade de compreensão oral. Como há o envolvimento de duas áreas de grande 
importância para linguagem, era esperado que os comprometimentos fossem mais evidentes em relação às habilidades de nomeação, compreensão escrita e leitura. Quanto ao discurso desses pacientes, mais uma vez é importante ressaltar que o mesmo foi investigado apenas no requisito fluência, enfatizando a ausência de esforço articulatório concomitante a uma boa velocidade de emissão, não sendo evidenciada a qualidade do discurso e presença de parafasias, hesitações ou perífrases. Por esta razão, da mesma forma como foi observado entre os indivíduos com lesão na região temporal, não se pode descartar a possibilidade do discurso estar comprometido no seu conteúdo sem alterar a fluência.

A literatura descreve, como no trabalho de Damasio (1998), que lesões nesta área podem apresentar quadros de linguagem semelhantes à afasia de condução. Este quadro de afasia estaria relacionado com lesão em região perisylviana esquerda, o córtex auditivo primário, e a região ao redor desta área, e em grau variável com a ínsula, a substância branca subcortical e o giro supramarginal. $\mathrm{Na}$ afasia de condução as características mais evidentes são: a preservação da compreensão, um discurso fluente, porém marcado de hesitações com parafasias fonêmicas, e uma nítida dificuldade de repetição. Entretanto, no presente estudo, não foi observada uma correlação significativa da repetição com a lesão na região têmporo-parietal (correlação negativa muito baixa -0,03), assim como se evidenciou uma correlação positiva com a compreensão oral $(0,24)$.

Diante dos resultados do grupo de pacientes com lesão em região têmporo-parietal, a classificação mais aproximada seria a afasia transcortical sensorial, pois há uma diminuição da compreensão oral, mas não há o comprometimento da repetição. Essas características das habilidades da linguagem, neste grupo, podem corresponder a um menor envolvimento da área de Wernicke nesses pacientes. Desta forma, a região que circunda esta área, com comprometimento da região parietal, traria como resultado uma afasia transcortical sensorial como descreve Hillis (2007) e Vendrell (2001).

Já no grupo de indivíduos portadores de lesão em território parietal/ parieto-occipital foi observada uma correlação positiva, apesar de discreta, com a repetição $(0,14)$, assim como com a compreensão oral $(0,66)$. Esse é um resultado intrigante, visto que tal comprometimento não pode ser caracterizado, como descreve Vendrell (2001) como se tratando de uma afasia de condução, pelo fato da compreensão oral não estar preservada, nem como uma afasia transcortical sensorial, pelo fato da repetição estar comprometida, apesar de discretamente. É importante, ainda, ressaltar que a compreensão escrita não esteve comprometida no grupo em questão, uma vez que se observou uma correlação negativa com esta habilidade $(-0,10)$.

Outro aspecto importante foi o fato de habilidade da leitura não apresentar uma correlação positiva tanto com o grupo têmporo-parietal, quanto com o grupo com lesão na região parietal/parieto-occipital, pois os resultados mostram uma correlação negativa nos dois grupos. Era de se esperar um maior envolvimento desta função lingüística com estas áreas lesionadas.

\section{Considerações Finais}

O presente estudo revelou a ocorrência de correlações importantes entre as habilidades de linguagem e as áreas cerebrais esquerdas comprometidas por lesão vascular. Entretanto, a análise MDS revelou que tais correlações nem sempre foram fiéis com a determinação dos locais de lesão defendidas no passado.

Neste mesmo sentido, Basso (2000), em uma revisão sobre estudos que reforçam essas diferenças e questionam a localização específica das funções da linguagem, defende a hipótese de que redes neurais complexas, assim como sistemas cognitivos complexos, são recrutadas para tarefas aparentemente simples da linguagem tais como a nomeação de figuras.

No mesmo sentido, Fridriksson, Morrow-Odom, Moser, Fridriksson e Baylis (2006) propõem que, para as tarefas de linguagem, há o recrutamento e a sobreposição de várias redes neurais ao longo das diversas áreas cerebrais. Jodzio et al. (2003) acrescentam que os afasiologistas modernos não concordam totalmente com a exata correspondência de funções especificas com áreas cerebrais especificas. Para esses autores, as alterações das funções superiores não são unicamente decorrentes de lesão em determinadas áreas, ditas como responsáveis por estas funções, mas podem ser definidas pela desconexão provocadas por lesões em áreas distantes.

Esta distribuição das habilidades da linguagem nas respectivas áreas lesadas enfatiza cada vez mais o dinamismo das funções cognitivas que apresenta variações de alterações determinadas por lesões semelhantes, como descreve Perea-Bartolomé (2001). Estas diferentes manifestações podem ser determinadas pelas diversas influências que o cérebro recebe como, a idade, o sexo, a dominância manual, as influências sócio-culturais, a aquisição de novas habilidades que proporcionam ao cérebro humano características individuais e que estas podem resultar em uma estruturação própria da linguagem corroborando com os estudos de Castro-Caldas, Petersson, Reis, Stone-Elander e Ingvar (1998), Mesulam (2000), Pereira et al. (2003).

Assim, apesar de a análise MDS trazer uma boa contribuição à discussão do tema, determinados aspectos permanecem fontes de inquietude para as pesquisas na área. Limitações quanto ao alcance de algumas provas para avaliar habilidades lingüísticas de sujeitos cérebro lesionados, assim como a influência de aspectos sócioculturais merecem destaque e carecem de melhores investigações. 


\section{Referências}

Alexander, M. P., Naeser, M. A., \& Palumbo, C. (1990). Broca’s area aphasias: Aphasia after lesions including the frontal operculum. Neurology, 40(2), 353-362.

Barraquer, L. B. (2008). Las estereotipias de los afásicos. Su génesis y su diversidad. Neurología, 23(3), 197-199.

Basso, A. (2000). The aphasias: Fall and renaissance of the neurological model? Brain and Language, 71, 15-17.

Castro-Caldas, A., Petersson, K. M., Reis, A., Stone-Elander, S., \& Ingvar, M. (1998). The illiterate brain. Learning to read and write during childhood influences the functional organization of the adult brain. Brain, 121(6), 1053-1063.

Damasio, H. (1998). Neuroanatomical correlates of the aphasias. In Acquired aphasia ( $3^{\text {rd }}$ ed., pp. 43-70). San Diego, CA: Academic Press.

DeLeon, J., Gottesman, R. F., Kleinman, J. T., Newhart, M., Davis, C., Heidler-Gary, J., et al. (2007). Neural regions essential for distinct cognitive processes underlying picture naming. Brain, 130(5), 1408-1422.

Demonet, J. F., Thierry, G., \& Cardebat, D. (2005). Renewal of the neurophysiology of language: Functional neuroimaging. Physiological Reviews, 85(1), 49-95.

Dronkers, N. F. (2000). The pursuit of brain-language relationships. Brain and Language, 71(1), 59-61.

Dronkers, N., \& Ogar, J. (2004). Brain areas involved i speech production. Brain, 127(Pt. 7), 1461-1462.

Dronkers, N. F., Plaisant, O., Iba-Zizen, M. T., \& Cabanis, E. A. (2007). Paul Broca's historic cases: High resolution MR imaging of the brains of Leborgne and Lelong. Brain, 130, 1432-1441.

Faroqi-Shah, Y., \& Thompson, C. K. (2007). Verb inflections in agrammatic aphasia: Encoding of tense features. Journal Memory Language, 56, 129-151.

Fridriksson, J., Morrow-Odom, L., Moser, D., Fridriksson, A., \& Baylis, G. (2006). Neural recruitment associated with anomia treatment in aphasia. Neuroimage, 32(3), 14031412.

Gold, B. T., Balota, D. A., Kirchhoff, B. A., \& Buckner, R. L. (2005). Common and dissociable activation. Patterns associated with controlled semantic and phonological processing: Evidence from fMRI adaptation. Cerebral Cortex, 15, 14381450 .

Goodglass, H., \& Kaplan, E. (1984). The assessment of aphasia and related disorders. Philadelphia, PA: Williams \& Williams.

Guttman, L. (1968). A general nonmetric technique for finding the smallest co-ordinate space for a configuration. Psychometrika, 33, 469-506.

Hillis, A. E. (2007). Aphasia: Progress in the last quarter of a century. Neurology, 69, 200-213.

Jodzio, K., Gasecki, D., Drumm, D. A., Lass, P., \& Nyka, W. (2003). Neuroanatomical correlates of the post-stroke aphasias studied with cerebral blood flow SPECT scanning. Medical Science Monitor, 9(3), MT32-MT41.

Knaus, T. A., Bollich, A. M., Corey, D. M., Lemen, L. C., \& Foundas, A. L. (2006). Variability in perisylvian brain anatomy in healthy adults. Brain and Language, 97, 219-232.

Lecours, A. R., Mehler, J., Parente, M. A., Caldeira, A., Cary, L., Castro, M. J., et al. (1987). Illiteracy and brain damage 1. Aphasia testing in culturally contrasted populations (control subjects). Neuropsychologia, 25(1B), 231-245.
Lecours, A. R., Mehler, J., Parente, M. A., Aguiar, L. R., da Silva, A. B., Caetano, M., et al. (1987). Illiteracy and brain damage - 2. Manifestations of unilateral neglect in testing “Auditory comprehension” With iconographic materials. Brain and Cognition, 6, 243-265.

Lecours, A. R., Mehler, J., Parente, M. A., Beltrami, M. C., Canossa de Tolipan, L., Cary, L., et al. (1988). Illiteracy and brain damage - 3: A contribution to the study of speech and language disorders in illiterates with unilateral brain damage (initial testing). Neuropsychologia, 26(4), 575-589.

Mansur, L. L. (2003). O estudo da linguagem e do cérebro a partir de lesões em adultos. In S. C. O. Limongi, Fonoaudiologia: Informação para formação (pp. 123-137). Rio de Janeiro, RJ: Guanabara Koogan.

Mesulam, M. (2000). Brain, mind, and the evolution of connectivity. Brain and Cognition, 42(1), 4-6.

Miceli, G., Silveri, C., Romani, C., \& Caramazza, A. (1989). Variation in the pattern of omissions and substitutions of grammatical morphemes in the spontaneous speech of socalled agrammatic patients. Brain and Language, 36, 447-492.

Murdoch, B. E. (1997). Síndromes de afasia de Boston e Luria. In B. E. Murdoch, Desenvolvimento da fala e distúrbios da linguagem (pp. 53-83). Rio de Janeiro, RJ: Revinter.

Murtha, S., Chertkow, H., Beauregard, M., \& Evans, A. (1999). The neural substrate of picture naming. Journal of Cognitive Neuroscience, 11, 399-423.

Perea-Bartolomé, M. (2001). Classificación del síndrome afásico. Revista Neurología, 32(5), 483-488.

Pereira, J. R., Reis, A. M., \& Magalhães, Z. (2003). Neuroanatomia funcional: Anatomia das áreas activáveis nos usuais paradigmas em ressonância magnética funcional. Acta Médica Portuguesa, 16, 107-116.

Shapiro, K. A., Môo, L. R., \& Caramazza, A. (2006). Cortical signatures of noun and verb production. Proceedings of the National Academy of Science, 103(5), 1644-1649.

Soares, E. C. S., \& Ortiz, K. Z. (2008). Influence of brain lesion and educational background on language tests in aphasic subjects. Dementia \& Neuropsychologia, 2(4), 321-327.

Vendrell, J. M. (2001). Aphasia: Semiology and clinical patterns. Revista Neurología, 32(10), 980-986.

Young, F. W. (1987). Multidimensional scaling: History, theory and applications. Hillsdale, NJ: Erlbaum.
Recebido: 06/05/2009 $1^{a}$ revisão: 04/09/2009 $2^{a}$ revisão: 07/01/2010 $3^{a}$ revisão: $29 / 03 / 2010$ Aceite final: 04/06/2010 